\title{
AVALIAÇÃO DO CLIMA DE SEGURANÇA DO PACIENTE ENTRE PROFISSIONAIS DE ENFERMAGEM
}

\author{
ASSESSMENT OF PATIENT SAFETY CLIMATE AMONG \\ NURSING PROFESSIONALS
}

\section{EVALUACIÓN DEL CLIMA DE SEGURIDAD DEL PACIENTE ENTRE PROFESIONALES DE ENFERMERÍA}

\author{
Andreia Castro de Sousa França* \\ Wellyson da Cunha Araújo Firmo** \\ Wochimann de Melo Lima Pinto*** \\ Maria Edileuza Soares Moura****
}

\section{RESUMO}

Objetivo: Avaliar o clima de segurança sob a perspectiva dos profissionais da equipe de enfermagem, atuantes nas clínicas médica e cirúrgica de dois hospitais do interior do estado do Maranhão, Brasil, após realização de uma capacitação sobre segurança do paciente. Material e método: Estudo com abordagem quantitativa do tipo pós-intervenção que recrutou 105 profissionais de enfermagem atuantes nas clínicas médica e cirúrgica de dois hospitais. Após uma capacitação sobre segurança do paciente aplicou o Safety Attitudes Questionnaire (SAQ). Resultados: Os escores por cada domínio do SAQ nas clínicas médica e cirúrgica em Açailândia e Imperatriz respectivamente foram: clima de trabalho em equipe, 54,67 e 63,43; clima de segurança, 57,25 e 53,99; satisfação no trabalho, 81,67 e 80,59; percepção de estresse, 46,40 e 66,51; percepção da gerência do hospital, 57,27 e 54,03; condiçóes de trabalho, 57,27 e 51,02. Em uma escala com escore máximo de 100 e aceitável de 75, o escore final do SAQ foi 58,75 em Açailândia e 60,43 em Imperatriz, que são considerados baixos. Conclusão: Identificaram-se graves deficiências no clima de segurança com escores abaixo de 75 e a necessidade da implantaçáo, eficaz, dos protocolos de segurança do paciente.

Palavras-chave: Segurança do Paciente; Serviços de Saúde; Gestão da Segurança.

\footnotetext{
*Farmacêutica. Mestre em Biodiversidade, Ambiente e Saúde pela Universidade Estadual do Maranhão e Docente do Instituto Federal do Maranhão, Santa Inês, Brasil. Email: andreiacastrosousafranca1@gmail.com. Autor da correspondência.

**Farmacêutico. Doutor em Biodiversidade e Biotecnologia pela Universidade Federal do Maranhão e Docente da Universidade Ceuma, São Luís, Brasil. Email: well_firmo@hotmail.com

***Enfermeira. Mestre em Administração pelo Sistema Integrado de Minas Gerais e Docente na Faculdade Santa Terezinha e Faculdade Pitágoras, São Luís, Brasil. Email: wochilima@gmail.com

****Enfermeira. Doutora em Medicina Tropical pela Universidade Federal de Goiás e Docente da Universidade Estadual do Maranhão, Caxias, Brasil. Email: mariaedileuzasoares@hotmail.com
} 


\section{ABSTRACT}

Objective: To evaluate the safety climate from the perspective of nursing team professionals working in the medical and surgical clinics of two hospitals in the interior of the state of Maranhão, Brazil, after conducting a training on patient safety. Materials and methods: Study with quantitative post-intervention approach that recruited 105 nursing professionals working in the medical and surgical clinics of two hospitals. After a training on patient safety, the Safety Attitudes Questionnaire (SAQ) was applied. Results: The scores for each SAQ domain in the medical and surgical clinics in Açailândia and Imperatriz, respectively, were: teamwork climate, 54.67 and 63.43; safety climate, 57.25 and 53.99; job satisfaction, 81.67 and 80.59; stress perception, 46.40 and 66.51; perception of hospital management, 57.27 and 54.03; working conditions, 57.27 and 51.02. On a scale with a maximum score of 100 and an acceptable score of 75, the final score of the SAQ was 58.75 in Açailândia and 60.43 in Imperatriz, which are considered low. Conclusion: Serious deficiencies in the safety climate were identified with scores below 75 and the need for effective implementation of patient safety protocols.

Keywords: Patient Safety; Health Services; Safety Management.

\section{RESUMEN}

Objetivo: Evaluar el clima de seguridad desde la perspectiva de los profesionales del equipo de enfermería que trabajan en las clínicas médicas y quirúrgicas de dos hospitales en el interior del estado de Maranhao, Brasil, después de realizar un entrenamiento sobre la seguridad del paciente. Material y método: Estudio con enfoque cuantitativo post-intervención que reclutó a 105 profesionales de enfermería que trabajaban en las clínicas médicas y quirúrgicas de dos hospitales. Después de una capacitación sobre la seguridad del paciente se aplicó el Cuestionario de Actitudes de Seguridad (SAQ). Resultados: Las puntuaciones de cada dominio de SAQ en las clínicas médicas y quirúrgicas de Açailândia e Imperatriz, respectivamente, fueron: clima de trabajo en equipo, 54,67 y 63,43; clima de seguridad, 57,25 y 53,99; satisfacción laboral, 81,67 y 80,59; percepción del estrés, 46,40 y 66,51; percepción de la gestión hospitalaria, 57,27 y 54,03; condiciones de trabajo, 57,27 y 51,02. En una escala con una puntuación máxima de 100 y una puntuación aceptable de 75, la puntuación final de la SAQ fue de 58,75 en Açailândia y 60,43 en Imperatriz, que se consideran bajas. Conclusión: Se identificaron deficiencias graves en el clima de seguridad con puntuaciones inferiores a 75 y la necesidad de aplicar eficazmente los protocolos de seguridad del paciente.

Palabras clave: Seguridad del paciente; Servicios de salud; Gestión de Seguridad.

Fecha recepción: 03/01/2019

Fecha aceptación: 09/09/2019

\section{INTRODUÇÁO}

A segurança do paciente é definida pela diminuição do risco de danos desnecessários associados à assistência à saúde até o mínimo aceitável. Esse "mínimo aceitável" refere-se ao possível diante do conhecimento atual, dos recursos disponíveis e do contexto em que a assistência foi realizada frente ao risco de não tratamento ou de outro tratamento. Sendo assim, a segurança do paciente consiste em reduzir atos inseguros nos processos assistenciais e usar as melhores práticas descritas de forma a alcançar os melhores resultados possíveis para o paciente $^{(1)}$.
A divulgação de um relatório do Institute of Medicine (IOM) To Err is Human em 1999 deu relevância à temática segurança do paciente. Esse documento se baseou em duas pesquisas de avaliação da incidência de eventos adversos (EAs) em revisóes retrospectivas de prontuários, estas pesquisas foram realizadas em hospitais americanos de Nova York, Utah e Colorado nas quais foram analisados prontuários de 30.121 internaçóes e identificados sérios prejuízos iatrogênicos ocorridos em 3,7\% das internações $(6,5 \%$ dos quais provocaram disfunçóes permanentes e 13,6\% envolveram a morte do paciente). Com base nestes resultados, estimou-se que os danos haviam contribuído para a ocorrência 
de 180.000 óbitos por ano naquele país ${ }^{(2-4)}$.

Avaliando-se os custos com o tratamento das consequências dos EAs, estudos em serviços de saúde europeus revelaram gastos que variam de $1 \mathrm{a}$ 1.4 bilhōes de Euros, como também foi observado aumento da permanência hospitalar na ordem de 1,08 dias, apontando para a necessidade de investimento em estratégias de prevençãa ${ }^{(5,6)}$.

Em 2004, a World Health Organization (WHO) corroborando com a preocupação da situação, criou a World Alliance for Patient Safety. Os objetivos dessa proposta (que passou a chamar-se Patient Safety Program) foram, entre outros, organizar uma taxonomia sobre segurança do paciente e propor medidas para reduzir os riscos e aplacar os $\mathrm{EAs} s^{(1,7,8)}$.

No Brasil, as investigaçóes revelaram que a avaliação do clima de segurança do paciente em hospitais ainda é deficiente e carente de melhorias em vários aspectos que envolvem a promoção da saúde $^{(9,10)}$ descritivo e com abordagem quantitativa. Foi utilizado o SafetyAttitudes Questionnaire(SAQ). Um estudo pioneiro realizado em três hospitais no Rio de Janeiro, Brasil, buscou identificar a incidência de EAs, os autores levantaram que a incidência de EA foi de 7,6\%, e, destes, $67 \%$ foram classificados como evitáveis. Esse estudo mostrou que os EAs mais frequentes foram as infecçóes associadas aos cuidados da saúde, como observado em outros países em desenvolvimento, representando 24,6\% dos EAs evitáveis, seguidas das complicaçóes cirúrgicas e/ou anestésicas, 20\%; atraso ou falha no diagnóstico e/ ou tratamento, 18,4\%; lesôes por pressão, 18,4\% e complicações na punção venosa, 7,7\%, sugerindo que os EAs evitáveis representem um sério risco para o cuidado hospitalar no Brasil, e que este cenário pode ser modificado por açóes que não requerem demasiada complexidade ${ }^{(11)}$.

Os EAs estão associados ao aumento da mobimortalidade hospitalar, do tempo de internação, do custo relacionado ao cuidado e risco aumentado para o óbito, sendo o ambiente dos cuidados críticos o local onde os riscos são mais altos ${ }^{(12,13)}$. No Brasil os EAs impactam negativamente o Sistema Único de Saúde por aumentarem o tempo de internação do paciente, ampliação de comorbidades e maiores impactos financeiros para o serviço público de saúde ${ }^{(14)}$.

Assim, partindo da definição de segurança do paciente como a 'redução, a um mínimo aceitável, do risco de dano desnecessário associado à atenção à saúde' infere-se a necessidade de também definir a cultura da segurança, ou seja, 'o conjunto de valores, atitudes, competências e comportamentos que determinam o comprometimento com a gestão da saúde e da segurança, substituindo a culpa e a punição pela oportunidade de aprender com as falhas e melhorar a atenção à saúde' ${ }^{(14)}$.

Autores da área de segurança do paciente defendem que a qualidade do cuidado em saúde pode ser alcançada com uma força de trabalho de enfermagem fortalecida, habilitada e comprometida. Assim, os profissionais de enfermagem despontam como peça fundamental para a concretização de um cuidado seguro, admitindo-se que é a categoria profissional mais numerosa dentro do setor saúde, dedica mais tempo ao cuidado direto ao paciente e realiza numerosos procedimentos neste, por consequência as chances de cometer um erro durante a assistência é maior ${ }^{(15,16)}$.

Nesse contexto, o Ministério da Saúde instituiu o Programa Nacional de Segurança do Paciente (PNSP), por meio da Portaria MS/GM no 529, de 1 de abril de 2013, com o objetivo geral de contribuir para a qualificação do cuidado em saúde, em todos os estabelecimentos de saúde do território nacional, quer sejam públicos, quer privados ${ }^{(8)}$. De acordo com esta diretriz, pesquisadores do estudo propuseram uma capacitação para os profissionais $\mathrm{da}$ enfermagem objetivando situar os mesmos quanto aos esforços do Ministério da Saúde em instalar o PNSP, bem como apresentar aspectos básicos de cada meta internacional de segurança do paciente.

Reconhecendo que a qualidade da equipe de enfermagem contribui, de forma efetiva e direta, para uma boa evoluçáo do quadro de saúde dos pacientes e seu contentamento diante dos cuidados recebidos e que essa qualidade está estreitamente relacionada com a segurança da assistência prestada por estes profissionais é que os objetivos deste estudo foram: Avaliar o clima de segurança dos pacientes sob a perspectiva dos profissionais da equipe de enfermagem, atuantes nas clínicas médica e cirúrgica de dois hospitais do interior do Maranhão utilizando-se Safety Attitudes Questionnaire (SAQ), traduzido e validado para a língua portuguesa, aplicado após um curso de capacitação sobre segurança do paciente ${ }^{(17)}$.

Esse estudo justifica-se pela magnitude de EAs relacionados à assistência à saúde, muitas vezes evitáveis, ao alto custo referente à permanência 
hospitalar devido a estas complicaçóes desnecessárias ao paciente, e também a falta de conhecimento relacionado ao tema segurança do paciente entre profissionais e a sociedade em geral.

\section{MATERIAL E MÉTODO}

Tipo de estudo: Estudo de abordagem quantitativa do tipo pós-intervenção.

Local do estudo: A área geográfica de abrangência desse estudo foram os municípios de Imperatriz e Açailândia, ambos pertencentes à Regiāo Metropolitana do Sudoeste do Maranhão, na regiáo Nordeste do Brasil.

Amostra: Composta por profissionais da equipe de enfermagem atuantes nas clínicas médica e cirúrgica que atenderam aos critérios de inclusão do estudo: pertencer à equipe de enfermagem (ser enfermeiro, técnico em enfermagem ou auxiliar de enfermagem); estar, pelo menos, há três meses trabalhando no setor; trabalhar, pelo menos, $20 \mathrm{~h}$ semanais no setor. Foi critério de exclusão: estar em férias e/ou licença no período de coleta de dados. Os dois hospitais possuíam 170 profissionais da enfermagem nas clínicas médica e cirúrgica: 135 de Imperatriz e 35 de Açailândia, sendo 130 auxiliares ou técnicos em enfermagem e 40 enfermeiros. Realizou-se o cálculo do tamanho da amostra para populaçóes finitas, tendo uma população total conhecida de 170 profissionais e estimou-se a amostra em 109 participantes (margem de erro tolerável de 5\% e nível de confiança de 95\%).

Capacitaçáo em segurança do paciente: Curso de qualificaçáo profissional sobre o Programa Nacional de Segurança do Paciente (PNSP) em forma de debates multiparticipativos sobre as seis metas internacionais de segurança do paciente, realizado nos dias $8,9,10,27,28,29,30$ de novembro de 2017 nos hospitais municipais de Açailândia e Imperatriz. A capacitação trouxe o tema aos profissionais de forma dinâmica e participativa, onde eles puderam expor suas ideias e opinióes acerca do assunto com a construção dos quadros de melhorias sugeridos pelos profissionais presentes, onde ficou claro que muita coisa precisa ser modificada em especial na área comportamental e estrutura física hospitalar, reafirmando a importância da participação dos mesmos no processo da implantaçáo dos protocolos de segurança do paciente.

\section{Coleta e instrumento para obtençáo dos dados:}

A coleta de dados ocorreu no período de 8 a 30 de novembro de 2017 posterior ao curso de capacitação em segurança do paciente. Como instrumento de coleta de dados, foi utilizado o Questionário de Atitudes de Segurança - Safety Attitudes Questionnaire (SAQ) validado e adaptado culturalmente para a realidade dos hospitais brasileiros $^{(17)}$.

Quanto ao conteúdo, este instrumento é composto por duas partes: A primeira contém 36 questóes, envolvendo a percepção sobre segurança do paciente. A segunda parte visa a coletar dados do profissional: cargo exercido, gênero, atuação principal e tempo de atuação.

Assim este instrumento mensura a percepção dos profissionais de saúde por meio de seis domínios:

1. Clima de Trabalho em Equipe: considerado como a qualidade do relacionamento e a colaboraçáo entre os membros de uma equipe (itens 1 a 6 );

2. Clima de Segurança: percepção dos profissionais quanto ao comprometimento organizacional para segurança do paciente (itens 7 a 13);

3. Satisfaçáo no Trabalho: visão positiva do local de trabalho (itens 15 a 19);

4. Percepçáo do Estresse: reconhecimento de quanto os fatores estressores podem influenciar na execução do trabalho (itens 20 a 23);

5. Percepçáo da Gerência: aprovação das ações da gerência ou administraçáo, tanto da unidade em que o profissional atua, quanto do hospital como um todo (itens 24 a 28) e

6. Condiçóes de Trabalho: percepção da qualidade do ambiente de trabalho (itens 29 a 32).

Entretanto, os itens 14, 33 a 36 não fazem parte de nenhum domínio no instrumento original, mas foi considerado na mensuração da média final do escore SAQ.

A resposta de cada questão seguirá a escala de cinco pontos de Likert: opçáo A- discorda totalmente (0), B- discorda parcialmente (25), C- neutro (50), D - concorda parcialmente (75), E- concorda totalmente (100) e X- não se aplica (0). O escore final do instrumento varia de 0 a 100 , no qual zero corresponde à pior percepção de atitudes de segurança pelos profissionais de saúde e 100, à melhor percepçáo. Foram considerados 
valores positivos quando o total do escore é igual a 75. Os escores foram contados da seguinte forma: as questōes foram ordenadas por domínios, assim, somam-se as respostas das questóes de cada domínio e divide-se pelo número de questóes de cada um.

Análises dos dados: Após a coleta dos questionários, os dados foram inseridos em um banco de dados eletrônico (Programa Excel da Microsoft ${ }^{\oplus}$ ) por meio de dupla digitação no intuito de validar o banco de dados. Já o processamento dos dados foi realizado pelo Statistical Package for Social Science (SPSS), versão 11.0.

Aspectos Éticos: O projeto foi submetido ao Comitê de Ética em Pesquisa (CEP), via Plataforma Brasil para análise do cumprimento da Portaria no 466/2012 que normatiza as pesquisas envolvendo seres humanos, foi aprovado pelo CEP da Universidade Estadual do Maranhão segundo o parecer de $\mathrm{n}^{\circ}$ 2.325.226, em 10 de outubro de 2017. Todos os participantes assinaram o Termo de Consentimento Livre e Esclarecido e tiveram garantido o sigilo de sua identidade tornando-os cientes do trabalho a ser desenvolvido.

\section{RESULTADOS}

Participaram um total de 105 profissionais, mas houve a necessidade de exclusão de 5 questionários pois os mesmos foram respondidos por profissionais que não se encaixavam no objetivo da pesquisa (pois o alvo eram profissionais de enfermagem), sendo estes, 2 farmacêuticos e 3 assistentes sociais, permanecendo para a contabilização dos resultados as entrevistas de 100 profissionais da enfermagem das clínicas médica e cirúrgica, sendo 33 de Açailândia e 67 de Imperatriz. O sexo feminino predominou nos dois hospitais com respectivos 87,9\% em Açailândia e 91\% em Imperatriz (Tabela 1). O tempo de atuação da maioria dos profissionais foi de 3 a 10 anos de experiência, 63,6\% em Açailândia e 68,6\% em Imperatriz.

Tabela 1. Caracterizaçáo dos participantes do estudo segundo gênero, cargo e tempo de especialidade $(\mathrm{N}=100)$. Açailândia e Imperatriz, Maranhão, 2017.

\begin{tabular}{|c|c|c|c|}
\hline \multirow{2}{*}{ Variáveis } & & \multicolumn{2}{|c|}{ Frequência } \\
\hline & & $\mathbf{n}$ & $\%$ \\
\hline \multicolumn{4}{|l|}{ Açailândia } \\
\hline \multirow[t]{2}{*}{ Gênero } & Homem & 4 & 12,12 \\
\hline & Mulher & 29 & 87,9 \\
\hline \multirow[t]{2}{*}{ Cargo } & Auxiliar/Técnico de enfermagem & 22 & 66,66 \\
\hline & Enfermeiro (a) & 11 & 33,33 \\
\hline \multirow[t]{7}{*}{ Tempo de atuação } & Menos de 6 meses & 0 & 0 \\
\hline & 6 a 11 meses & 1 & 3,03 \\
\hline & 1 a 2 anos & 1 & 3,03 \\
\hline & 3 a 4 anos & 5 & 15,15 \\
\hline & 5 a 10 anos & 16 & 48,48 \\
\hline & 11 a 20 anos & 4 & 12,12 \\
\hline & 21 anos ou mais & 6 & 18,18 \\
\hline \multicolumn{4}{|l|}{ Imperatriz } \\
\hline \multirow[t]{2}{*}{ Gênero } & Homem & 6 & 8,95 \\
\hline & Mulher & 61 & 91 \\
\hline \multirow[t]{2}{*}{ Cargo } & Auxiliar/Técnico de enfermagem & 44 & 65,67 \\
\hline & Enfermeiro (a) & 23 & 34,32 \\
\hline \multirow[t]{7}{*}{ Tempo de atuação } & Menos de 6 meses & 1 & 1,49 \\
\hline & 6 a 11 meses & 3 & 4,48 \\
\hline & 1 a 2 anos & 5 & 7,46 \\
\hline & 3 a 4 anos & 10 & 14,93 \\
\hline & 5 a 10 anos & 36 & 53,73 \\
\hline & 11 a 20 anos & 7 & 10,45 \\
\hline & 21 anos ou mais & 5 & 7,46 \\
\hline
\end{tabular}


Com este estudo observou-se que o clima de segurança do paciente náo foi considerado favorável, pois o escore final do SAQ foi $58,75 \mathrm{em}$ Açailândia e 60,43 em Imperatriz (Tabela 2).

No que diz respeito aos escores por cada domínio das clínicas médica e cirúrgica em Açailândia e Imperatriz os domínios clima de trabalho em equipe, clima de segurança, percepçáo do estresse, percepção da gerência e condições de trabalho apresentaram escores não satisfatórios (abaixo de 75). Nos dois hospitais o domínio Satisfação no Trabalho obteve média de escore acima de 75, critério para satisfatório na avaliação do domínio (Tabela 3).

Tabela 2. Avaliação dos escores do Questionário de Atitudes de Segurança, segundo os domínios nos municípios de Açailândia e Imperatriz, Maranhão, 2017.

\begin{tabular}{lccccc}
\hline Domínios & Mínimo & Máximo & Mediana & Média & $\begin{array}{l}\text { Desvio } \\
\text { Padráo }\end{array}$ \\
\hline Açailândia & \multicolumn{7}{c}{} & & \\
\hline Clima de trabalho em equipe & 8,33 & 95,83 & 54,17 & 54,67 & 18,65 \\
Clima de segurança & 25 & 89,29 & 60,71 & 57,25 & 15,91 \\
Satisfação no trabalho & 50 & 100 & 85 & 81,67 & 13,09 \\
Percepção do estresse & 0 & 100 & 50 & 46,4 & 29,02 \\
Percepção da gerência & 20 & 100 & 50 & 57,27 & 21,86 \\
Condiçóes de trabalho & 6,25 & 100 & 43,75 & 57,27 & 26,23 \\
Q14,33 a a36 & 10 & 95 & 65 & 59,39 & 20,22 \\
\hline Escore geral & $\mathbf{3 7 , 5}$ & $\mathbf{9 0 , 2 8}$ & $\mathbf{5 6 , 9 4}$ & $\mathbf{5 8 , 7 5}$ & $\mathbf{1 1 , 3 9}$ \\
\hline Imperatriz & & & & & \\
\hline Clima de trabalho em equipe & 16,67 & 100 & 62,5 & 63,43 & 16,38 \\
Clima de segurança & 10,71 & 89,29 & 53,57 & 53,99 & 15,27 \\
Satisfação no trabalho & 20 & 100 & 85 & 80,59 & 16,88 \\
Percepção do estresse & 0 & 100 & 68,75 & 66,51 & 24,68 \\
Percepçáo da gerência & 0 & 90 & 55 & 54,03 & 20,96 \\
Condiçóes de trabalho & 0 & 100 & 50 & 51,02 & 30,03 \\
Q14,33 a a36 & 0 & 95 & 60 & 54,77 & 20,69 \\
\hline Escore geral & $\mathbf{2 1 , 5 3}$ & $\mathbf{8 8 , 8 9}$ & $\mathbf{6 1 , 1 1}$ & $\mathbf{6 0 , 4 3}$ & $\mathbf{1 2 , 3 9}$ \\
\hline
\end{tabular}

\section{DISCUSSÃO}

Uma análise sócio demográfica dos profissionais de enfermagem no Brasil, constatou que a equipe de enfermagem, a qual congrega enfermeiros, auxiliares e técnicos de enfermagem somam mais de um milhão e oitocentos mil trabalhadores, constituindo cerca de $50 \%$ da força de trabalho que atua no setor saúde ${ }^{(18)}$. Assim, a sensibilização dos profissionais de enfermagem para o compromisso com a segurança do paciente aparece como um alicerce para o fortalecimento do cuidado seguro.
A enfermagem, no espaço de oferta de cuidados ao paciente, se constitui a maior força de trabalho e, em sua maioria, atua como protagonista na assistência direta, permanecendo mais tempo com os pacientes, desenvolvendo açóes de numerosos procedimentos, tornando-se mais suscetível a cometer erros. Em contrapartida, alguns estudos apontam que a enfermagem éa categoria profissional diretamente mais envolvida com a segurança do paciente, destacando que conhecer os erros pode ser o ponto de partida para a implementaçáo de medidas preventivas ${ }^{(19)}$. 


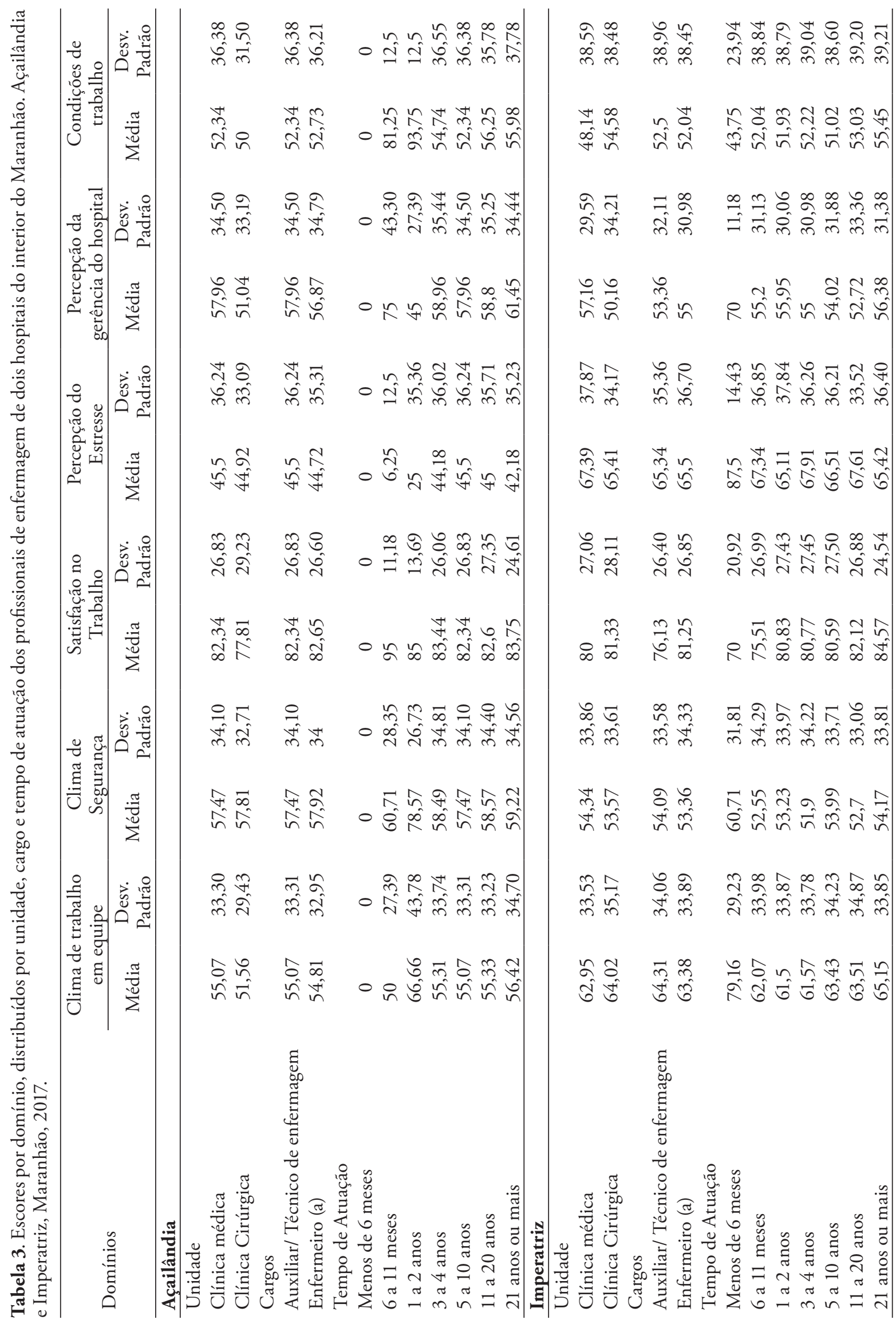


Os resultados obtidos pós-intervenção demonstrou que em geral, o clima de segurança do paciente entre os profissionais de enfermagem em estudo foi desfavorável. Dados atuais sobre clima de segurança apontam que as precárias condiçóes de trabalho, dinâmica de trabalho estressante, escassez de recursos materiais e demanda crescente por serviços de saúde apontam para uma avaliação pouco favorável (média do escore final do SAQ menor que 75$)^{(17,20)}$.

Os itens gênero, tempo de atuação e categoria profissional se assemelham a outros estudos em que predominaram trabalhadores do sexo feminino pertencentes à enfermagem, com cinco a 20 anos de experiência ${ }^{(10,21-24)}$.

Em conformidade com os resultados deste estudo, pesquisas nacionais observaram que a percepção quanto ao clima de segurança também pouco variou, de acordo com cada domínio, local/ unidade (clínica médica ou cirúrgica), categoria profissional e tempo de atuação clínicas médicas e cirúrgicas. Ao analisar as médias do SAQ total por categorias profissionais notou que prevalecerem baixos escores entre a maioria dos participantes, indicando que as duas clínicas possuem deficiências semelhantes, que necessitam de melhorias ${ }^{(10,21)}$.

O domínio satisfação no trabalho apresentou maior escore entre os profissionais do estudo. A aplicação do SAQ em unidades de terapia intensiva e em clínicas médica e cirúrgica constatou resultado similar entre os profissionais de enfermagem ${ }^{(20,25}$, 26). A boa percepção dos profissionais quanto a este domínio, foi um fator considerado positivo, uma vez que a satisfação do profissional com o trabalho está diretamente relacionada com a qualidade da assistência prestada ${ }^{(10)}$.

Em relação aos domínios, percepção da gerência e clima de trabalho em equipe resultados semelhantes foram encontrados, em uma pesquisa nacional a "Percepção da gerência do hospital" foi avaliada com escore igual a 40,9 e o "Clima de trabalho em equipe" 69,4 foi considerado baixo ${ }^{(21)}$. Em outro estudo nacional, o domínio Percepção da Gerência do hospital e da unidade foi o domínio que apresentou menores valores entre os profissionais de enfermagem ${ }^{(26)}$. A Percepção da Gerência pelo profissional é um fator importante para a garantia da segurança do paciente, uma vez que este domínio reflete a concordância do profissional quanto às ações da gerência ou da administração do hospital e da unidade relacionadas à segurança do paciente ${ }^{(10)}$.

Em consonância com o resultado para domínio "Condiçôes de trabalho" que foi considerado baixo e os itens isolados 33 a 35 os quais tratam do relacionamento profissional entre enfermeiros, médicos e farmacêuticos (apresentou escore desfavorável) e o item 36 (escore muito baixo) que discorre sobre comunicação entre os profissionais. Estudo realizado em um hospital universitário em Florianópolis (SC) identificou o escore para condições de trabalho foi 40 , e segundo os autores, resultado este devido aos itens isolados, que há graves falhas na comunicação entre setores ${ }^{(27)}$.

Destacou-se o domínio referente à satisfação no trabalho que atingiu porcentagem maior que $75 \%$, significando que mesmo diante de vários problemas existentes nos locais de trabalho os profissionais estão satisfeitos com sua profissão, e isso é o primeiro passo para serem condescendentes para as mudanças necessárias tanto na organização hospitalar, quanto na cultura de segurança a ser desenvolvida por eles.

O tipo de intervenção pode também ser classificado como profilático ou terapêutico (pré e pós intervenção). Como limitações aponta-se a impossibilidade de aleatorização e de manipulação de determinadas variáveis, deve-se ainda ser levada em consideração a questão ética, que envolve a manipulação de determinadas variáveis e as dificuldades de realizar experimentação em alguns serviços de saúde. Ressalta-se ainda a possibilidade de efeito Hawthorne, ou seja, as respostas dos participantes serem modificadas somente por se saberem participantes de um estudo ${ }^{(28)}$. Outra limitação do estudo pós-intervenção (o que não acontece no estudo pré-intervenção) seria dar informações a respeito das perguntas que seriam feitas no questionário da pesquisa; vale destacar que o instrumento de coleta de dados náo apresentou questionamentos relacionados ao conteúdo da capacitação realizada neste estudo e sim sobre domínios específicos acerca do clima de segurança do paciente não sendo, por tanto, uma forma de avaliar o aprendizado do profissional e sim as condições reais vividas por esses em relação à segurança do paciente no ambiente hospitalar.

Esforços coletivos precisam ser empregados para que o fortalecimento da cultura de segurança do paciente torne-se realidade. Nesse sentido, 
os resultados podem ser utilizados como ponto de partida para que gestores, em parceria com os Núcleos de Segurança do Paciente, possam elaborar um plano para a melhoria do clima de segurança, direcionar açóes que poderão ser realizadas com a finalidade de reduzir os fatores facilitadores de erros, bem como alcançar uma assistência de qualidade e um cuidado seguro.

\section{REFERÊNCIAS}

1. World Health Organization. World Alliance for Patient Safety, Taxonomy: The Conceptual Framework for the International Classification for Patient Safety: final technical report. Genebra: WHO; 2009.

2. Belela ASC, Peterlini MAS, Pedreira MLG. Revelação da ocorrência de erro de medicação em unidade de cuidados intensivos pediátricos. Rev. Bras. Ter. Intensiva. 2010; 22 (3): 257-263.

3. Gawande AA, Thomas EJ, Zinner MJ, Brennan TA. The incidence and nature of surgical adverse events in Colorado and Utah in 1992. Surgery. 1999; 126 (1): 66-75.

4. Kohn LT, Corrigan JM, Donaldson MS. To err is human. Washington, DC: National Academy Press; 2000. 312 p.

5. Allue N, Chiarello P, Bernal Delgado E, Castells X, Giraldo P, Martínez N, et al. Assessing the economic impact of adverse events in Spanish hospitals by using administrative data. Gac Sanit. 2014;28 (1):48-54.

6. Magdelijns FJ, Stassen PM, Stehouwer CD, Pijpers E. Direct health care costs of hospital admissions due to adverse events in the Netherlands. Eur J Public Health. 2014; 24(6): 1028-1033.

7. World Health Organization. World Alliance for Patient Safety: Forward Programme 2005. Geneva: WHO; 2004. 33 p.

8. Ministério da Saúde Brasil. Gabinete do Ministro. Portaria MS/GM No 529, de 1 de Abril de 2013. Institui o Programa Nacional de Segurança do Paciente (PNSP). Diário Oficial da União; Brasília, DF, 2013 Abr 12; Seção 1. p. 43-44.

9. Luiz RB, Simóes ALA, Barichello E, Barbosa $\mathrm{MH}$. Fatores associados ao clima de segurança do paciente em um hospital de ensino. Rer Lat Am Enfermagem. 2015; 23(5): 880-887.

\section{CONCLUSÁO}

Com os resultados desta pesquisa realizada após intervenção conclui-se que o clima de segurança do paciente segundo a perspectiva dos profissionais de enfermagem das clínicas médica e cirúrgica dos hospitais municipais de Açailândia e Imperatriz possui uma percepção desfavorável, principalmente nos domínios clima de segurança e percepção da gerência.

10. Rigobello MCG, Carvalho REFL, Cassiani SHB, Galon T, Capucho HC, Deus NN. Clima de segurança do paciente: percepção dos profissionais de enfermagem. Acta Paul Enferm. 2012; 25(5): 728735 .

11. Mendes W, Martins M, Rozenfeld S, Travassos C. The assessment of adverse events in hospitals in Brazil. Int J Qual Health Care. 2009; 21(4): 279284.

12. Pedrosa TMG, Couto RC. Erros e eventos adversos na assistência médico-hospitalar. Revista Médica de Minas Gerais, Belo Horizonte. 2014; 24 (2): 216222.

13. Roque KE, Tonini T, Melo ECP. Eventos adversos na unidade de terapia intensiva: impacto na mortalidade e no tempo de internação em um estudo prospectivo. Cad. Saúde Pública [Internet]. 2016 Oct [citado 2017 jun 16]; 32(10): 1-15. Disponível em: http://www.scielo.br/pdf/csp/ v32n10/pt_1678-4464-csp-32-10-e00081815.pdf

14. Ministério da Saúde Brasil. Agência Nacional de Vigilância Sanitária-ANVISA. Resolução de Diretoria Colegiada- RDC No 36, de 25 de julho de 2013. Institui açôes para a segurança do paciente em serviços de saúde e dá outras providências. Diário Oficial da União; Brasília, DF, 2013; Seção 1, p. 32.

15. Ministério da Saúde Brasil. Agência Nacional de Vigilância Sanitária-ANVISA. Medidas de prevenção de infecção relacionada à assistência à saúde. [Internet]. Brasil: ANVISA; 2017 [citado 2017 oct 06]. 122 p. Disponível em: https:// www20.anvisa.gov.br/segurancadopaciente/index. $\mathrm{php} /$ publicacoes/item/caderno-5

16. Nunes FDO, Barros LAA, Azevedo RM, Paiva SS. Segurança do paciente: como a enfermagem vem contribuindo para a questáo? J. res. fundam. care. online [Internet]. 2014 [citado 2017 jun 16]; 6(2): 841-47. Disponível em: http://www.seer. unirio.br/index.php/cuidadofundamental/article/ 
view/3007/pdf_1296

17. Carvalho REFL, Cassiani SHB. Questionário Atitudes de Segurança: adaptaçáo transcultural do Safety Attitudes Questionnaire - Short Form 2006 para o Brasil. Rev Lat Am Enfermagem. 2012; 20 (3): $75-582$.

18. Machado MH, Aguiar Filho W, Lacerda WF, Oliveira E, Lemos W, Wermelinger $\mathrm{M}$ et al. Características gerais da Enfermagem: o perfil sócio demográfico. Enferm. Foco. 2015; 6(1/4): 11-17.

19. Fragata JIG. A segurança dos doentes - Indicador de qualidade em saúde. Rev Port Clin Geral. 2010; 26: 564-570.

20. Cauduro FLF, Sarquis LM, Sarquis LMM, Cruz EDA. Cultura de segurança entre profissionais de centro cirúrgico. Cogitare Enferm. 2015; 20 (1): 128-37.

21. Marinho MM, Radünz V, Tourinho FSV, Rosa LM, Misiak M. Intervençôes educativas e seu impacto na cultura de segurança: uma revisão integrativa. Enferm. Foco. 2016; 7(2): 72-77.

22. Ministério da Saúde Brasil. Agência Nacional de Vigilância Sanitária-ANVISA. Assistência segura: uma reflexão teórica aplicada à prática. Brasil: ANVISA; 2013. 172 p.

23. Carvalho REFL, Arruda LP, Nascimento NKP, Sampaio RL, Cavalcante MLSN, Costa ACP. Assessment of the culture of safety in public hospitals in Brazil. Rev Lat Am Enfermagem
[Internet]. 2017 [citado 2018 mai 05]; 25: 1-8. Available from: http://www.scielo.br/pdf/rlae/ v25/0104-1169-rlae-25-e2849.pdf

24. Poley MJ, Van der Starre C, Van den Bos A, Van Dijk M, Tibboel D. Patient safety culture in a Dutch pediatric surgical intensive care unit: An evaluation using the Safety Attitudes Questionnaire. Pediatric Crit Care Med. 2011; 12(6): 310-316.

25. Carney BT, West P, Neily J, Mills PD, Bagian JP. Differences in nurse and surgeon perceptions of teamwork: implications for use of a briefing checklist in the OR. OR. AORN J. 2010; 91(6): 722-29.

26. Patterson PD, Huang DT, Fairbanks RJ, Wang HE. The emergency medical services Safety Attitudes Questionnaire. Am J Med Qual. 2010; 25(2):109115.

27. Santiago THR, Turrini RNT. Cultura e clima organizacional para a segurança do paciente em Unidades de Terapia Intensiva. Rev Esc Enferm USP. 2015; 49: 123-130.

28. Kypri K, McCambridge J, Wilson A, Attia J, Sheeran P, Bowe $S$ et al. Effects of Study Design and Allocation on participant behaviour-ESDA: study protocol for a randomized controlled trial. Trials [Internet]. 2011 [citado 2015 Maio 26];12(1):42. Available from: http://www.trialsjournal.com/ content/pdf/1745-6215-12-42.pdf 